\title{
Mathematica 4.0
}

\section{von Michael Joswig}

„Mathematica is the world's only fully integrated environment for technical computing." So beginnt das fast 1500 Seiten starke Handbuch der im Sommer 1999 erschienenen Version 4.0.

Auch wenn diese Selbsteinschätzung etwas unbescheiden klingt: Die Integration unterschiedlicher Aspekte aus dem Spannungsfeld zwischen Computer und Mathematik ist tatsächlich bereits seit der ersten Version von 1988 Mathematicas Domäne. Die graphische Oberfläche, die Benutzerführung, die Verknüpfung von Berechnungen mit Graphik und Sound setzen unverändert den Standard für die gesamte Branche.

Das Programm hat nicht nur Mathematiker als Zielgruppe im Auge. Mathematica hat eine Menge zu bieten für diejenigen, die mittelbar mit Mathematik in Kontakt kommen. Dazu gehören Naturwissenschaftler und Ingenieure, aber auch Volks- und Betriebswirte, denen Excel zu beschränkt ist.

Was gibt es für die Mathematiker Neues in der aktuellen Version?

Rein äußerlich bleibt fast alles beim Alten. Die wesentlichen Veränderungen sind nicht direkt sichtbar. Insgesamt ist Mathematica 4.0 spürbar schneller als seine Vorgänger. Dies gilt insbesondere für die $\mathrm{Nu}-$ merik, aber auch für Teile der Ganzzahlarithmetik. ${ }^{1}$

Es gibt aber auch ganz neue Fähigkeiten. Zu nennen sind beispielsweise die symbolische Laplace- und Fouriertransformation. Ein besonders interessantes neues Merkmal ist noch als „experimental“ deklariert (und vermutlich deswegen nur spärlich ${ }^{2}$ dokumentiert): Mathematica 4.0 hat etwas zur Quantorenelimination über $\mathbb{R}$ gelernt. Prinzipiell erschließt sich damit ein ganzer Kontinent von möglichen Anwendungen: von der nicht-linearen oder sogar der symbolischen Optimierung über die Robotik bis zur Konstruktion von Realisierungsräumen von Polytopen und dergleichen mehr, was sich mithilfe von semialgebraischen Varietäten beschreiben lässt. Mathematica beherrscht hierzu mehrere Verfahren. Implementiert ist Collins' Methode der zylindrischen algebraischen Dekomposition (CAD). Entscheidender Nachteil von CAD ist jedoch die außerordentliche Komplexität: vor allem eine größere Anzahl von Variablen macht ihr zu schaffen ${ }^{3}$. Daher wird CAD in der Mathematica-Implementierung unterstützt durch die alternativen Algorithmen von Weißpfenning und Loos, die auf Polynome mit geringem Grad aber vielen Variablen spezialisiert sind.

Insbesondere CAD ist geeignet, auch komplizierte algebraische Kurven und Flächen topologisch richtig darstellen zu helfen. Dies lässt entsprechende Graphikfunktionen um so schmerzlicher vermissen. ${ }^{4}$ Kompliziertere Flächen sind in Mathematica nach wie vor nur zu erschließen, wenn man eine passende Parametrisierung vorweisen kann.

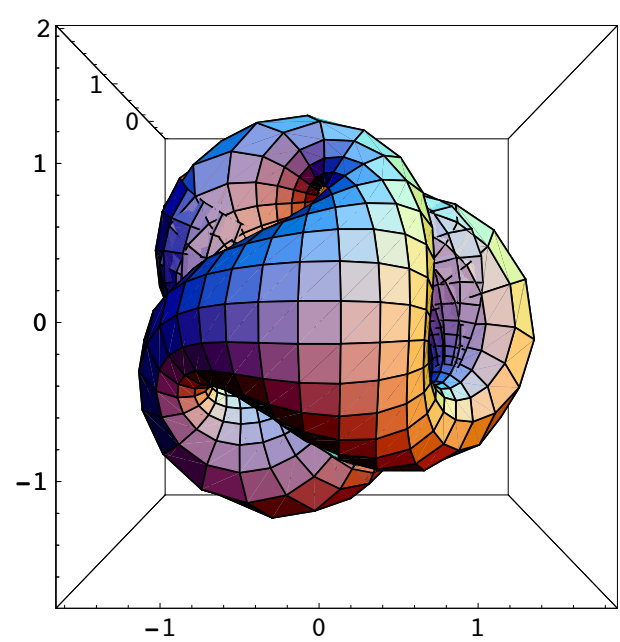

Die Boysche Fläche stellt eine Immersion der reellen projektiven Ebene in den $\mathbb{R}^{3}$ dar. Ein Modell steht vor der Bibliothek des Mathematischen Forschungsinstituts in Oberwolfach. Diese Ansicht „von oben“ wurde mit Mathematica produziert. Die Parametrisierung ist [1] entnommen.

Bemerkenswert ist nach wie vor die Mathematica eigene Programmiersprache. Während es im weiten Feld der mathematischen Software immer noch üblich ist, sich mit Sprachen zu begnügen, die im weitesten Sinne dem Algoltyp zuzuordnen sind, hat Mathematica von Beginn an versucht, Mathematik mit modernen Sprachkonzepten zu verbinden. Im wesentlichen steht das Programm damit bis heute allein. Grundsätzlich orientiert sich die Sprache an funktionalen Paradigmen, die um einzelne imperative Konzepte erweitert sind. Gekrönt wird das ganze von einem speziellen Pattern-Matching-Mechanismus.

1 Für einen Einblick in die Leistungsfähigkeit von Mathematica und anderen Computer-Algebra-Systemen siehe [2].

2 Nur in der Online-Version des Handbuchs; und auch dort recht unübersichtlich.

3 Bereits die meisten Probleme mit mehr als vier Variablen gelten hierbei als schwer.

4 Maple verfügt zu diesem Zweck über Funktionen implicitplot und implicitplot3d. Allerdings verlässt sich Maple dabei vollständig auf numerische Methoden. 


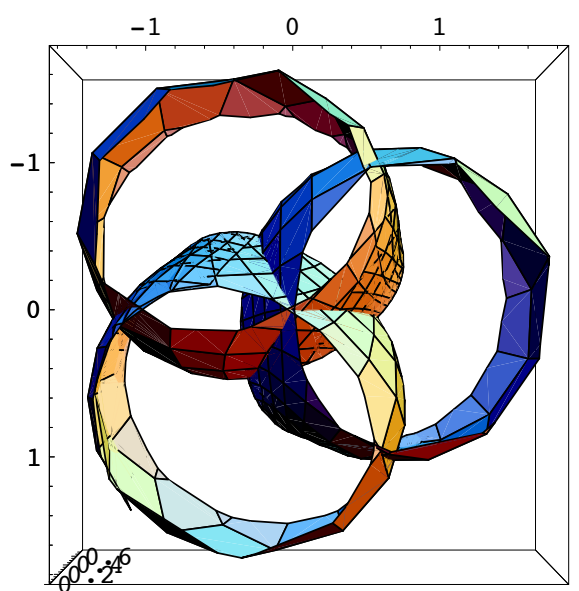

Schnitt der Boyschen Fläche. Blickrichtung ,von unten“. In der Mitte sieht man den Tripelpunkt.

Mathematica gehört unverändert zu den besten Produkten im Bereich mathematischer Software. Doch vor der Anschaffung der neuen Version 4.0 sollte man sich individuell überlegen, ob man die neuen Features wirklich benötigt. In vielen Fällen ist die alte Version völlig ausreichend.
(Mathematica 4.0 wird von Wolfram Research produziert und in Deutschland vertrieben durch Mathemas, c/o Carsten Herrmann, Königsbergerstr. 97, 24161 Altenholz, http://www.mathemas.de. Der aktuelle Preis für die kommerzielle Nutzung beträgt DM 3998. Es werden aber diverse, zum Teil erhebliche, Rabatte für Bildungseinrichtungen etc. gewährt, z. B. beträgt der Studentenpreis DM 264. Alle Preise variieren mit dem Kurs des britischen Pfund.)

\section{Literaturverzeichnis}

[1] Hermann Karcher und Ulrich Pinkall: Die Boysche Fläche in Oberwolfach, Mitteilungen der DMV, Heft $1 / 97,45-47$.

[2] Heinz Kredel: http://krum.rz.uni-mannheim.de/ cabench/diractiv.html, 19. Juli 1999.

[3] Wolfram Research: http://www.mathematica.com

\section{Adresse des Autors}

Dr. Michael Joswig

FB Mathematik, Sekr. 7-1

Technische Universität Berlin

Straße des 17. Juni 136

10623 Berlin

joswig@math.tu-berlin.de

\section{mathemas http://www.mathemas.de}

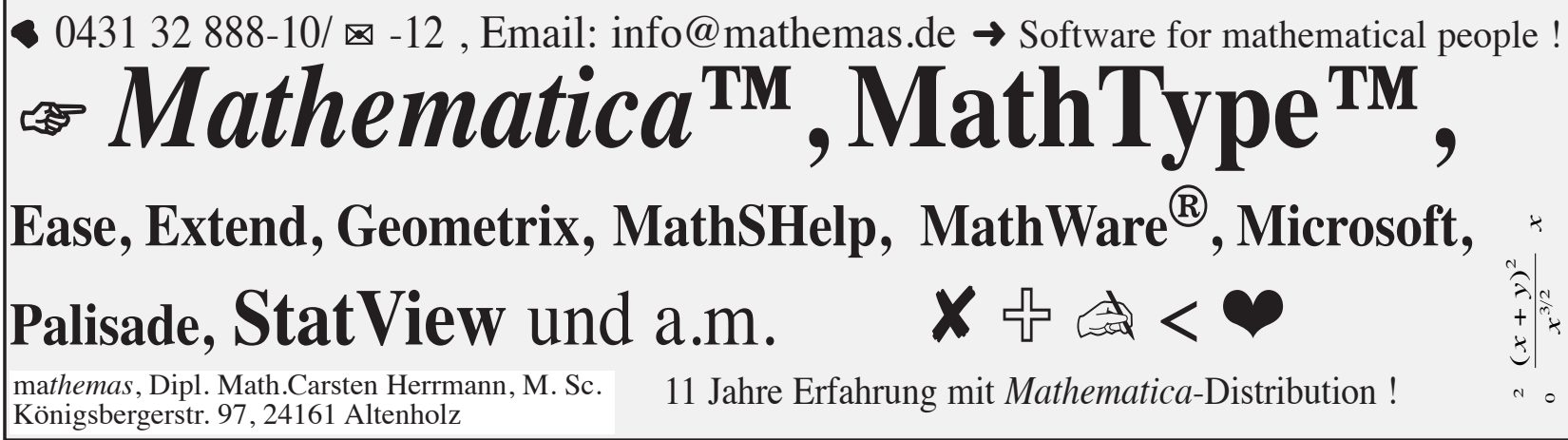

